\title{
Temporal structure coding with and without awareness
}

\author{
N. Faivre ${ }^{\mathrm{a}, *}$, C. Koch $^{\mathrm{a}, \mathrm{b}}$ \\ ${ }^{\text {a } C o m p u t a t i o n}$ and Neural Systems, California Institute of Technology, Pasadena, CA, USA \\ ${ }^{\mathrm{b}}$ Allen Institute for Brain Science, Seattle, WA, USA
}

\section{A R T I C L E I N F O}

\section{Article history:}

Received 30 July 2013

Revised 7 January 2014

Accepted 27 February 2014

\section{Keywords:}

Consciousness

Temporal integration

Continuous flash suppression

Apparent motion

Biological motion

\begin{abstract}
A B S T R A C T
In order to interpret a constantly changing environment, visual events far apart in space and time must be integrated into a unified percept. While spatial properties of invisible signals are known to be encoded without awareness, the fate of temporal properties remains largely unknown. Here, we probed temporal integration for two distinct motion stimuli that were either visible or rendered invisible using continuous flash suppression. We found that when invisible, both the direction of apparent motion and the gender of point-light walkers were processed only when defined across short time periods (i.e., respectively $100 \mathrm{~ms}$ and $1000 \mathrm{~ms}$ ). This limitation was not observed under full visibility. These similar findings at two different hierarchical levels of processing suggest that temporal integration windows shrink in the absence of perceptual awareness. We discuss this phenomenon as a key prediction of the global neuronal workspace and the information integration theories of consciousness.
\end{abstract}

(c) 2014 Elsevier B.V. All rights reserved.

\section{Introduction}

When one is immersed in a visual environment, the optical flow is constantly changing as objects move and as one moves in relation to these objects, defining temporal structures (Blake \& Lee, 2005). Processing these dynamic cues implies the existence of temporal integration windows within which different aspects of the signal changing over time (e.g., successive positions of a point of light) are integrated into a unified percept (e.g., a moving point). The duration of integration windows can be defined as the maximal delay between two events for which a response differs from the summed responses associated with each event. This reflects the fact that visual features conveyed by temporal structures are not defined at any one point in time, but are emergent properties of temporal

\footnotetext{
* Corresponding author. Address: California Institute of Technology, Biology Division, Mail Code 216-76, 1200 E. California Blvd., Pasadena, CA 91125, USA. Tel.: +1 6262008402.

E-mail address: nfaivre@caltech.edu (N. Faivre).
}

integration. We followed this logic and measured temporal integration for apparent and biological motion. In apparent motion, light points alternating between non-contiguous spatial locations are not perceived as flickering, but as moving back and forth (Anstis, 1980). For instance, if two vertically aligned dots are presented alternatively on either side of a virtual rectangle, with a delay less than the temporal integration window, one does not perceive two pairs of dots flickering out of phase, but two single dots moving back and forth along the rectangle's horizontal axis. Perceiving such motion implies that the location of the first point remains available until the second appears (i.e., the signal must be integrated over this duration). In addition, the correspondence between dots must be established, so that the same identity is attributed to two dots from pairs seen at different locations and times (Dawson, 1991). In the given example, a strong bias for horizontal correspondence between the dots creates the percept of horizontal motion, although the display could be as well interpreted as two dots moving along the diagonals of the rectangle. 
Involving more complex motion processing, point-light walkers are points of light representing the structural arrangement of the main joints of a walker across time (Johansson, 1973). Despite their simplicity, one can perceive the gender of point-light walkers, both using structural cues (e.g., the shoulder-hip ratio, larger for males) and kinematic cues (e.g., the lateral body sway, more pronounced for females) (Mather \& Murdoch, 1994; Troje, 2002). While structural cues are encoded by the spatial relationships between light points, and are therefore available at each individual image frame, kinematic cues only arise when integrating information across frames. Hence, one can study spatial and temporal integration from structurally and kinematically defined point-light walkers, respectively.

Static spatial features that are defined at any point in time (e.g., location, orientation, color) are known to be encoded and integrated into objects even when these are perceptually invisible (see Lin \& He, 2009 for a review). Yet, the integration of such invisible static features into dynamic features (e.g., motion speed, direction, synchrony) has not hitherto been addressed.

In order to assess the role of awareness during temporal integration, we used apparent and biological motion that were either fully visible or rendered invisible by continuous flash suppression (CFS). In CFS, a stream of salient patterns flashed to the dominant eye renders the signal presented to the non-dominant eye invisible (Tsuchiya \& Koch, 2005; Tsuchiya, Koch, Gilroy, \& Blake, 2006). Relying on previous results (Anstis, Giaschi, \& Cogan, 1985; Blake, Ahlström, \& Alais, 1999; Geisler, 1999; Jordan, Fallah, \& Stoner, 2006; Kruse, Stadler, \& Wehner, 1986; Rajimehr, Vaziri-Pashkam, Afraz, \& Esteky, 2004; Ramachandran, 1975; Troje, Sadr, Geyer, \& Nakayama, 2006; Wiesenfelder \& Blake, 1991), we assumed that in the presence of temporal integration, being exposed to apparent motion with a particular direction or a point-light walker with kinematic features (hereafter the adaptor) would bias the way subsequent ambiguous motion is perceived (i.e., apparent motion with ambiguous direction, or point-light walkers with ambiguous gender). By varying parametrically the kinematics and visibility of apparent and biological motion adaptors, we could quantitatively estimate the extent of temporal integration with and without awareness.

\section{Methods}

\subsection{Participants}

Healthy volunteers with normal or corrected-to-normal visual acuity were recruited from the campus student population (age range: 18-30). Twenty participants were included in the apparent motion experiments ( 8 in the visible condition, 12 in the invisible condition, 3 females in each group). Fifty-eight participants were included in the biological motion experiments (visible condition: 9 for structural and kinematic point-light walkers (4 females), 8 for structural point-light walkers ( 4 females), 7 for kinematic point-light walkers (4 females); invisible condition: 7 for structural and kinematic point-light walkers ( 5 females), 8 for structural point-light walkers (5 females), 9 for kinematic point-light walkers (5 females); 10 for visible and invisible slow kinematic point-light walkers ( 1 female)). Subjects were naive to the purpose of these experiments, and gave informed written consent. All experiments conformed to Institutional Guidelines and to the Declaration of Helsinki.

\subsection{Apparatus}

Stimuli were presented using Matlab and the Psychophysics toolbox (Brainard, 1997; Pelli, 1997). Participants' heads were stabilized using a chinrest located $57 \mathrm{~cm}$ away from a 19" CRT screen (resolution $1024 \times 768$; $100 \mathrm{~Hz}$ refresh rate). A mirror stereoscope was used to present images separately to each eye.

\subsection{Continuous flash suppression}

A frame composed of textured black and white bars $\left(0.58^{\circ}\right.$ width $)$ was presented to each eye to facilitate stable fusion. Each trial started with a key press, once participants made sure that a fixation dot presented to the dominant eye $\left(0.23^{\circ}\right.$ diameter $)$ was centered within a circle presented to the non-dominant eye $\left(1.16^{\circ}\right.$ diameter). CFS patterns consisted of arrays of 600 randomly generated disks (from $0.08^{\circ}$ to $1.16^{\circ}$ diameter) of different shades of gray flashed at $10 \mathrm{~Hz}$ to the dominant eye.

\subsection{Apparent motion}

\subsubsection{Stimuli}

Stimuli consisted of disks of $1.55^{\circ}$ diameter, 0.23 Michelson contrast, convoluted with a Gaussian alpha mask $\left(\right.$ sigma $\left.=0.96^{\circ}\right)$. In the adaptation phase, the illusion of horizontal apparent motion was produced by presenting these disks for $100 \mathrm{~ms}$ alternatively on the long edges of an imaginary rectangle, while vertical apparent motion was produced by presenting them on the short edges.

\subsubsection{Aspect ratio procedure}

Before the adaptation phase, the rectangle's aspect-ratio was adapted for each observer so that on average, durations of vertical and horizontal motion perception were equal (aspect ratio procedure, see Kohler, Haddad, Singer, \& Muckli, 2008). For this purpose, two disks were presented at a time, on diagonally opposite corners of an imaginary rectangle, leading to a bistable alternation of horizontal and vertical motion (motion quartet, see Kruse et al., 1986). First, a motion quartet with an aspect ratio of 1 was presented, $\left(8.7^{\circ}\right.$ horizontal and vertical distances between the disks), leading to unambiguous perception of vertical motion. Observers had to report the direction of the quartet motion continuously by holding one of two directional keys. After each period of $2 \mathrm{~s}$, the vertical distance was increased or decreased by $0.4^{\circ}$ in order to compensate for the dominant direction perceived during this time window. The optimal aspect ratio was chosen as the average of the vertical distances across ten perceptual reversals (i.e., switch from vertical to horizontal direction). On average, the vertical to horizontal average ratio was 
$1.40(S E=0.055)$, so that dots were situated on a rectangle with a constant horizontal distance of $8.7^{\circ}$, and an average vertical distance across subjects of $12.12^{\circ}$.

\subsubsection{Adaptation phase procedure}

Two pairs of disks were presented for $100 \mathrm{~ms}$ alternatively on the long edges (horizontal apparent motion), or on the short edges (vertical apparent motion) of the optimal rectangle defined above. An adaptor with four disks presented simultaneously was used as a baseline (no motion, flicker condition). In order to vary parametrically the temporal structure of apparent motion adaptors, 100, 400,800 , or $1200 \mathrm{~ms}$ blank periods were added between the onset of successive pairs of disks (i.e., inter-stimulus interval (ISI), see Fig. 1). Apparent motion adaptors were presented for $20 \mathrm{~s}$. This duration was determined from pilot experiments as the maximal duration allowing for invisibility under CFS. While the non-dominant eye always received the apparent motion adaptor, the dominant eye received either a static homogeneous gray background in

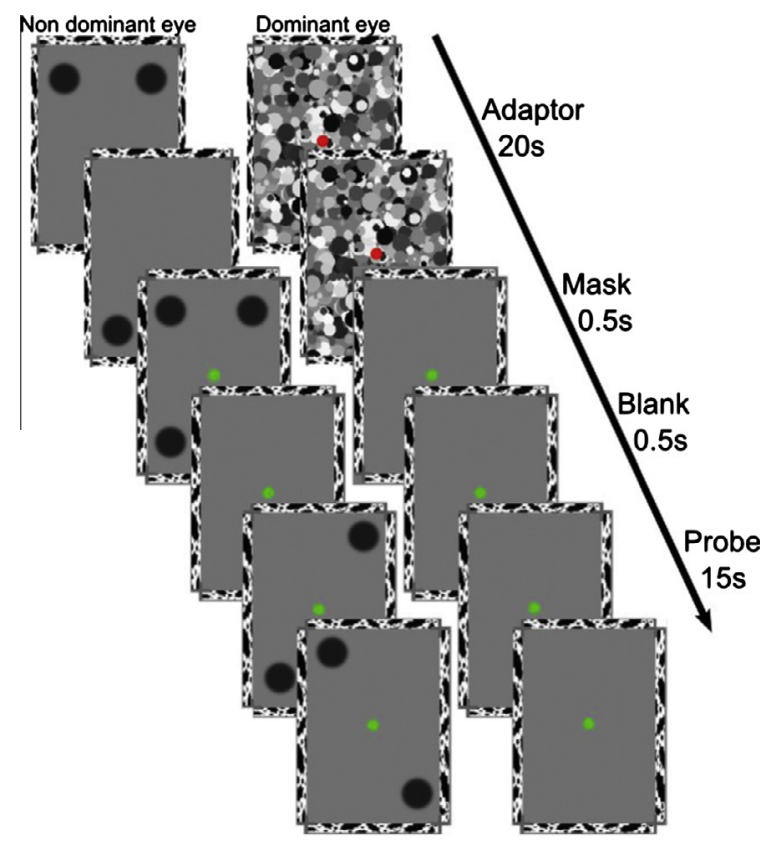

Fig. 1. Experimental paradigm for apparent motion. While the nondominant eye receives the adapting stimulus (the adaptor, here with a vertical direction), the dominant eye receives either a static homogeneous gray background (visible condition) or a stream of salient patterns (invisible condition, as depicted here). Observers had to indicate the adaptor's direction of motion by a key press (providing a measure for objective accuracy). They could provide an answer at any time during the $20 \mathrm{~s}$; we counted only the final one. Subsequently, the red fixation dot turned green, and a mask consisting of four simultaneous disks followed by a blank was presented, so that the last position of the adaptor could not influence subsequent events. Participants had to report continuously whether they perceived the probe as moving vertically or horizontally during $15 \mathrm{~s}$. In the invisible condition, each trial ended with a question asking participants if they had no perceptual experience of motion, a brief glimpse, an almost clear image, or a perfectly clear image of the adaptor (subjective visibility, see methods). (For interpretation of the references to color in this figure legend, the reader is referred to the web version of this article.) the visible condition, or a stream of CFS patterns in the invisible condition. At any point during the $20 \mathrm{~s}$ of display, participants had to indicate the adaptor's motion direction by pressing one of three keys (i.e., vertical motion, horizontal motion, or flicker). This provided an objective measure reflecting the participants' capacity to discriminate the direction of apparent motion (i.e., hereafter objective accuracy). Participants were asked to guess even though they could not see the adaptor. If several key presses occurred, the last one was considered as reflecting the participant's final decision. If no key press occured, the trial was rejected from further analysis, and participants were reminded to provide an answer in the next trials. After the adaptor, a 500 ms mask (i.e., four simultaneous disks) followed by a $500 \mathrm{~ms}$ blank were presented in order to reduce the influence of low-level similarities shared between adaptors and probes (i.e., so that the very last position of the adaptor's disks could not influence subsequent events). Motion quartet probes were presented during $15 \mathrm{~s}$ with a constant ISI of $200 \mathrm{~ms}$, allowing for several perceptual alternations to occur, and therefore have a continuous measure of adaptation. Participants reported whether they perceived vertical or horizontal motion of the probe during $15 \mathrm{~s}$, by holding continuously one of two directional keys. The experiment contained 72 trials and lasted around $1 \mathrm{~h}$. The different adaptors were presented in randomized order. In the invisible condition, participants were asked to indicate their subjective experience of the apparent motion adaptor (Ramsøy \& Overgaard, 2004): they pressed a key from 1 to 4 , corresponding respectively to "no perceptual experience", "a brief glimpse", "an almost clear image”, or "a perfectly clear image” of apparent motion.

\subsubsection{Statistical analysis}

Participants reported the direction of the probe by continuously holding one of two directional keys (i.e., vertical or horizontal motion). Key presses were recorded every $50 \mathrm{~ms}$. Based on this time series, we computed an adaptation index reflecting at each time stamp the congruency between the subjective report of the probe's direction and the direction of the adaptor: a value of -1 was assigned if both were identical (i.e., a participant reporting seeing the probe as moving vertically after a vertical adaptor), and 1 if they were opposite (i.e., a participant reporting seeing the probe as moving horizontally after a vertical adaptor). Time stamps for which no answer was provided were considered as missing values, so that the estimation of the adaptation index was not biased (e.g., during a switch between two key presses). We estimated potential perceptual biases for horizontal or vertical motion by measuring subjective reports of the probe's direction after a baseline adaptor containing no motion (i.e., flicker condition, see above). Such biases were subtracted from the adaptation index. Adaptation indices were computed individually for each ISI condition, and then compared at the group level using repeated measures analysis of variance. In the invisible condition, we discarded trials in which performance on the objective accuracy task was beyond $45 \%$ in a given subjective visibility category. In addition, three participants were excluded, as their global objective accuracy was significantly above chance-level 
( mean accuracy $=59 \%, S E=4.5, p=0.006$ ). Statistical analysis was performed using Matlab and $\mathrm{R}$.

\subsection{Biological motion}

\subsubsection{Stimuli}

Point-light walkers consisted in arrays of 15 dots, generated using the method developed by Troje (2002, 2008). Once recorded with a 9-camera motion capture system (Vicon 512, Oxford Metrics), the structure (i.e., defined by the dots' position) and kinematics (i.e., defined by the dots' motion) of 100 walkers (50 females) were projected in a parametric space using discrete Fourier decomposition. By convention, the average point-light walker was considered at the position 0 along an axis defining gender characteristics and consisted of 15 points. Position -1 corresponds to a walker 1 standard deviation (SD) away from the mean towards the female part of the axis, and a position of +1 refers to a walker 1 SD away from the mean towards the male part of the gender axis. Following previous results and taking into account biases in gender perception (Troje et al., 2006), we defined the female adaptor as -7 SD, and the male adaptor as +5 SD. Similarly, probes were defined at five equidistant steps from $-3 \mathrm{SD}$ (female) to +1 SD (male). Across the different experiments, adaptors and probes always contained the same gender information: either both structural and kinematic cues, structural cues only (i.e., structural cues of the specific gender, combined with kinematic cues of the average walker which are neutral in terms of gender), or kinematic cues only (i.e., structural cues of the average walker which are neutral in terms of gender, combined with kinematic cues of the specific gender, see Fig. 3b). The global size of adaptor and probes point-light walkers was $2.6^{\circ} \times 7.1^{\circ}$. Each of the 15 constituting dots was $0.23^{\circ}$ (Michelson contrast: 0.08 for adaptors, 0.36 for probes). In the fast kinematic
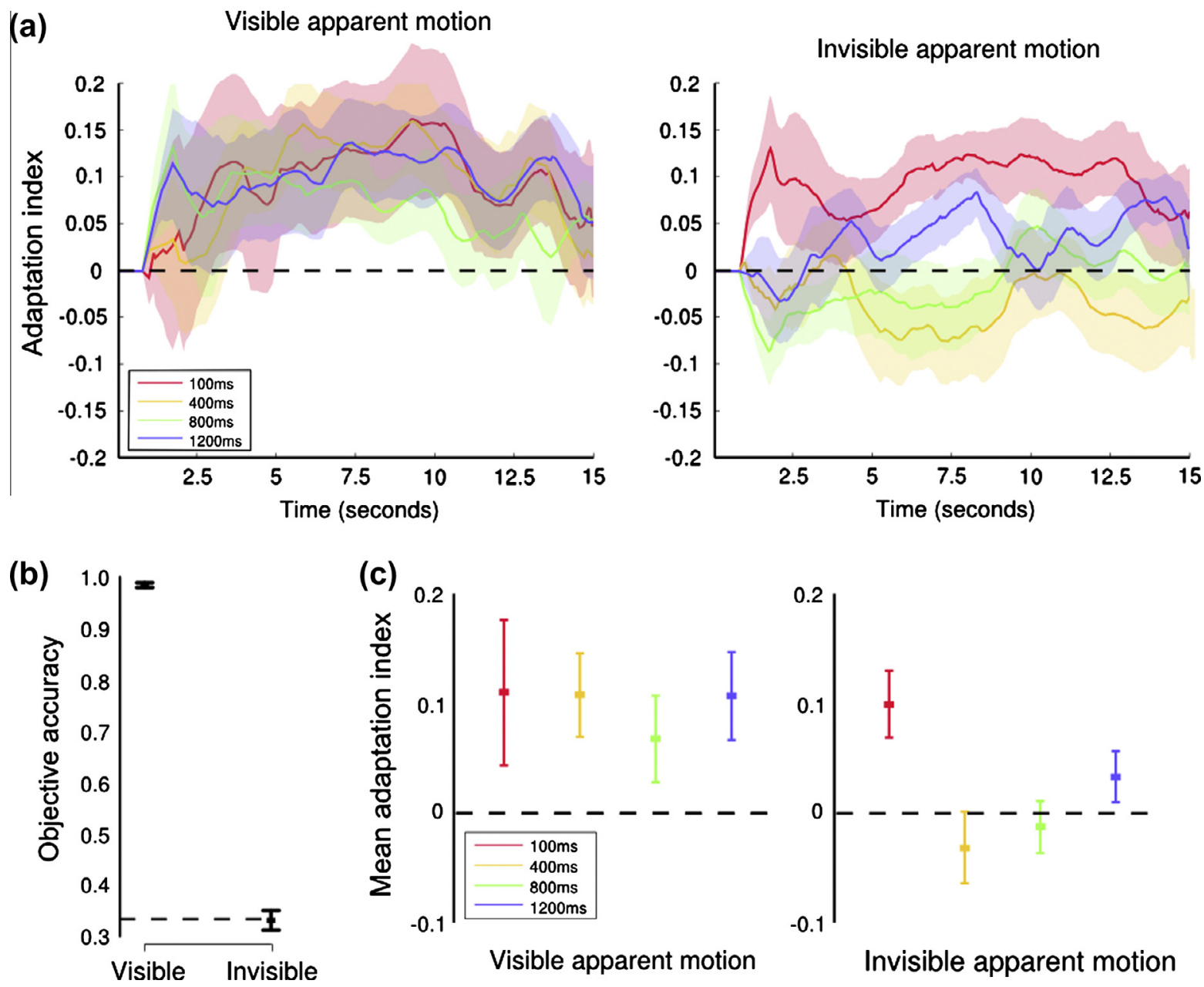

Fig. 2. Adaptation to visible and invisible apparent motion. (a) Time-course of the adaptation index in the visible (left panel) and invisible condition (right panel). Positive values on the vertical axis reflect the fraction of trials in which the probe was perceived as moving in the opposite direction than the adaptor. Red, yellow, green, and blue curves represent adaptors with an ISI of $100 \mathrm{~ms}, 400 \mathrm{~ms}, 800 \mathrm{~ms}$, and 1200 ms respectively. The colored bands correspond to one standard error of the mean (SEM). (b) Objective accuracy measures in the visible and invisible conditions after excluding irrelevant trials (see methods). Error bars stand for SEM. The dashed line corresponds to chance-level performance (i.e., 33\%, for 3 different adaptors). (c) Average of the adaptation index over the $15 \mathrm{~s}$ of probe display in the visible (left panel) and invisible condition (right panel). Error bars stand for SEM. (For interpretation of the references to color in this figure legend, the reader is referred to the web version of this article.) 
condition, the temporal sampling rate for the adaptor was $100 \mathrm{~Hz}$, so that a new array of 15 dots was presented every $10 \mathrm{~ms}$, and one walk cycle was $1 \mathrm{~s}$. In the slow kinematic condition, the temporal sampling rate for the adaptor was $33 \mathrm{~Hz}$, so that a new array of 15 dots was presented every $33 \mathrm{~ms}$, and one walk cycle was $3 \mathrm{~s}$. The temporal sampling rate for the probe was always $100 \mathrm{~Hz}$ (i.e., $1 \mathrm{~s}$ walk cycle).

\subsubsection{Adaptation phase procedure}

Point-light walker adaptors were presented for $3 \mathrm{~s}$, during which they underwent three walk cycles in the fast kinematic condition, or one walk cycle in the slow kinematic condition. The duration of $3 \mathrm{~s}$ was determined from pilot experiments as the maximal duration allowing for invisibility under CFS. After a blank of $200 \mathrm{~ms}$, a point-light walker probe was presented for $0.7 \mathrm{~s}$, during which it underwent a fraction of 0.7 walk cycle.

Unlike the apparent motion experiment, no mask was added between the adaptor and the probe, as they did not share the exact same position and kinematics (i.e., we could rule out low-level confounds, unlike in the apparent motion experiment). We aimed at maximizing our chance to detect adaptation rather than equating perfectly the two experiments, for which stimuli differ in many ways (see discussion). These parameters were chosen relying on the study by Troje et al. (2006). Adaptors were presented in a pseudo-randomized order, with no more than 3 successive repetitions of a same gender. The visible condition contained 100 trials, while the invisible conditions comprised 200 trials in order to increase signal to noise ratio.

\subsubsection{Statistical analysis}

We excluded trials for which the reaction time on the probe was below $200 \mathrm{~ms}$, or beyond 3 standard deviations from the mean (Whelan, 2010). The shape of the psychometric function was derived for each participant using a two-parameter logistic function in which the lower limit was fixed at 0 (i.e., for male probes at +1 SD) and the upper limit was fixed at 1 (i.e., for female probes at -3 SD). The midpoint of the logistic function across participants was used as a measure for the point of subjective equality. Effects were quantified as the average difference between points of subjective equality shift following male and female adaptors. The slope of the function at the midpoint was taken as a measure for the discriminability of male and female probes. No effect of discriminability was found in any of the conditions ( $p s>0.1$ ). Significance within a group of participants was tested using two-sample paired t-tests. Post-hoc comparisons between groups of participants were made using Welch two sample $t$-tests. Statistical analysis was performed using $\mathrm{R}$ ( $\mathrm{R}$ Foundation for Statistical Computing, 2013, Vienna, Austria).

\section{Results}

\subsection{Apparent motion}

On each trial, observers guessed the direction of the apparent motion adaptor (objective accuracy measure), and indicated how visible it was (subjective visibility measure, see Ramsøy \& Overgaard, 2004). The use of objective accuracy and subjective visibility measures allowed for a reliable estimation of awareness at the single trial level. Here, chance-level in objective accuracy was 33\%, as participants could choose between three alternatives: horizontal, vertical, or no apparent motion (see methods). In the visible condition (i.e., with no CFS), observers had a global objective accuracy of $98.4 \%(S E=0.47)$, revealing that they could detect the direction of apparent motion (a)

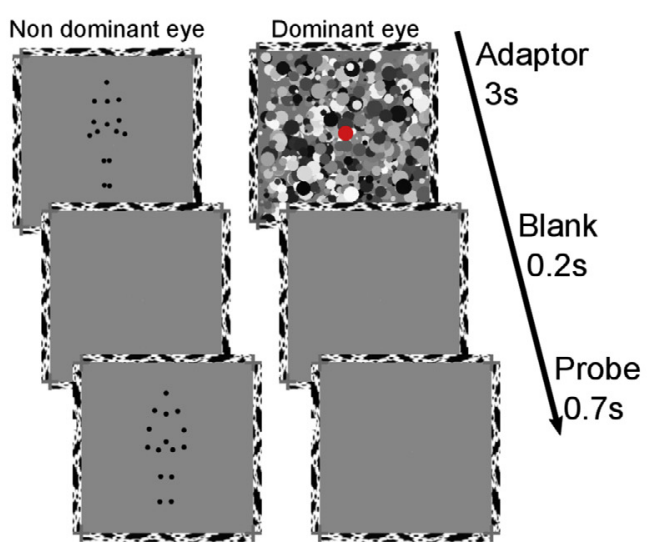

(b) Schematic drawings of PLW

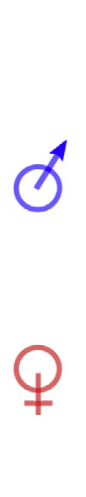

Structural
kinematic

Fig. 3. Experimental paradigm for biological motion. (a) While the non-dominant eye received the adaptor - 15 light points in each frame - during $3 \mathrm{~s}$, the dominant eye received either a gray background (visible condition), or a stream of salient patterns (invisible condition, as depicted here) masking the stimulus in the non-dominant eye. Following a blank of $0.2 \mathrm{~s}$, the probe was presented to the non-dominant eye for $0.7 \mathrm{~s}$. Observers had to categorize the probe's perceived gender, and then report their subjective percept of the adaptor, by indicating whether they have had no perceptual experience of the walker, a brief glimpse, an almost clear image, or a perfectly clear image of it. Finally, observers had to guess the adaptor's gender (objective accuracy measure). (b) Schematic description of male point-light walkers (top-row, in blue) and female point-light walkers (bottom-row, in red), either defined with structural (colored dots) and kinematic cues (colored arrows), structural cues only (colored dots with black arrows corresponding to neutral kinematic cues), or kinematic cues (black dots corresponding to neutral structural cues with colored arrows). For instance, note the bigger spacing between the two dots depicting the hips in female vs. male walkers in the structural condition, and their different trajectories in the kinematic condition. (For interpretation of the references to color in this figure legend, the reader is referred to the web version of this article.) 
in all ISI conditions (Fig. 2b). In the invisible condition (i.e., with CFS), observers reported having no experience of the adaptor in $66.2 \%$ ( $S E=9.72$ ) of trials. The average objective accuracy for these trials was $29.5 \%$ ( $S E=3.28$, with chance being $33.3 \%$, see Fig. $2 \mathrm{~b}$ ). Observers reported perceiving a brief glimpse in $20.6 \%$ ( $S E=6.09)$ of trials, with an objective accuracy of $40.3 \%$ ( $S E=7.76$ ), an almost clear image in $9.7 \%$ of trials $(S E=3.76)$, with an objective accuracy of $49.9 \%$ $(S E=8.43)$, and an absolute clear image in $3.5 \%$ of trials $(S E=2.73)$, with an objective accuracy of $39.7 \%$ $(S E=13.59)$. Subjective visibility and objective accuracy were related (linear regression analysis: adjusted $R^{2}=0.997, p=0.025$, the trials in which an "absolute clear image" was perceived were ignored because too rare for an accurate estimation of objective accuracy). After excluding cases of residual awareness (see methods), adaptors in the remaining trials were fully invisible (mean accuracy $=33.07 \%, p=0.97$ ). Importantly, we verified that ISI had no influence on subjective visibility (proportion of trials with no phenomenal experience of the adaptor: $F(3,33)=1.33, p=0.28)$. In addition, the interaction between ISI and objective accuracy was not significant $(F(3,33)=2.39, p=0.09)$. If anything, the trend for an interaction was driven by a surprising decrease of accuracy for $\mathrm{ISI}=400 \mathrm{~ms}(26 \%(S E=3.9)$ compared to $35 \%(S E=3.1)$ for ISI $=100 \mathrm{~ms}, 35 \%(S E=2.7)$ for $I S I=800 \mathrm{~ms}$, and $36 \%$ $(S E=3.4)$ for $I S I=1200 \mathrm{~ms}$, none of them being significantly higher than chance-level, with all $p s>0.4$ ). Overall, we can assume with reasonable confidence that objective accuracy was not consistently correlated with ISI.

We then estimated the adaptation index, reflecting whether the probe was perceived with the same or opposite direction compared to the adaptor (see methods). We found a significant adaptation effect from the adaptor (repeated measures ANOVA, adaptation index $=0.097$, $F(1,7)=8.08, p=0.025)$, with no interaction with ISI $(F(3,21)=0.31, p=0.82)$ (Fig. 2a and c). This reflects a repulsive, adaptation effect, whereby an ambiguous probe is perceived as horizontal after a vertical adaptor, and vice versa. Importantly, the absence of interaction with the ISI shows that this adaptation effect occurs irrespectively of the apparent motion kinematics. By contrast, in the invisible condition, we found an interaction of the adaptation index with ISI (one-way ANOVA, $F(3,33)=6.06, p=0.002$ ), revealing significant adaptation only for $I S I=100 \mathrm{~ms}$ (adaptation index $=0.10$, standard error $(S E)=0.031$, $t(11)=-3.38, p=0.007$; other ISI: all $p s>0.2$, see Fig. $2 \mathrm{c}$ ). Surprisingly, adaptation to visible and invisible apparent motion was of similar amplitude at ISI $=100 \mathrm{~ms}$ (Welch two sample $t$-test, $t(10.01)=0.13, p=0.90$, see discussion). Although average adaptation is far from being significant for ISI $=1200 \mathrm{~ms}(p>0.2)$, it is of note that the adaptation index diverged from baseline for a short duration around $8 \mathrm{~s}$ after adaptor offset (Fig. 2a, right panel, blue trace). This signal is hard to interpret, as we would expect adaptation to arise early after the adaptor offset, and to be sustained over time, similar to what happens in the $100 \mathrm{~ms}$ ISI condition. This divergence from baseline is therefore likely to reflect noisy signal fluctuations. In addition, besides the differences in terms of adaptation index described above, we found that visible and invisible adaptors had no influence on other aspects of the perceptual reports regarding the probe's direction (i.e., number and dynamics of perceptual switches between vertical and horizontal motion, $p s>0.1$ ). To sum up, these results suggest that adaptation to visible apparent motion is similar for ISIs between $100 \mathrm{~ms}$ and $1200 \mathrm{~ms}$, while adaptation to invisible apparent motion occurs only for a short ISI of $\sim 100 \mathrm{~ms}$.

\subsection{Biological motion}

We assessed the generality of our results on apparent motion by applying the same logic to the perception of biological motion. Across the different experiments, adaptor and probe point-light walkers contained either both structural and kinematic cues, structural cues only (i.e., genderspecific structural cues combined with neutral kinematic cues), or kinematic cues only (i.e., neutral structural cues combined with gender-specific kinematic cues) (Fig. 3). In each case, we computed the point of subjective equality at which a participant rated a probe to be equally likely male or female when preceded by a female or a male adaptor. Similar to the apparent motion experiment, we made sure that adaptors were invisible using both subjective and objective accuracy measures (Fig. 4a). In the visible condition (i.e., with no CFS), the proportion of trials in which subjects reported having an almost clear or perfectly clear image was $97.8 \%(S E=0.97)$ for structural and kinematic cues, $99.5 \%(S E=0.27)$ for structural cues only, and 92.4\% ( $S E=4.64)$ for kinematic cues only. In the invisible condition (with CFS), the same proportions fell respectively to $3.2 \%(S E=2.1), 1.4 \%(S E=0.64)$, and $7.2 \%(S E=5.40)$. In the visible condition, accuracy on the objective accuracy task for structural and kinematic cues was $97.8 \%$ $(S E=0.84), 98.2 \%(S E=0.77)$ for structural cues only, and $96.8 \%(S E=1.5)$ for kinematic cues only. In the invisible condition, we discarded trials in which performance on the objective accuracy task was beyond $60 \%$ in a given subjective visibility category. Seven participants were excluded from the invisible condition, as their global objective accuracy was significantly above chance-level (mean accuracy $=84 \%, S E=2.8, p=0.001$ ). In the remaining trials, performance on the objective accuracy task did not differ from chance level (50\%), as it fell respectively to $51.1 \%$ for structural and kinematic cues $(S E=1.27$, $t(6)=0.86, \quad p=0.42), 49.2 \%$ for structural cues only $(S E=0.96, t(7)=-0.78, p=0.46)$ and $51.2 \%$ for kinematic cues only $(S E=1.23, t(8)=1.01, p=0.34)$. Regression analyses confirmed the relation between subjective reports and objective accuracy in the invisible conditions for structural and kinematic cues (adjusted $\mathrm{R}^{2}=0.62, p=0.001$ ), structural cues (adjusted $\mathrm{R}^{2}=0.22, p=0.057$ ), and kinematic cues (adjusted $\mathrm{R}^{2}=0.66, p=0.001$ ). This confirmed that the gender of the visible adaptors was easily discriminable, and that CFS successfully masked salient and moving events like point-light walkers.

Adaptors containing both structural and kinematic cues induced priming both in the visible (effect $=0.66, S E=0.16$, two-sample paired $t$-test $t(8)=4.09, p=0.003)$, and invisible conditions (effect $=0.18, \quad S E=0.02, \quad t(6)=10.70$, $p=0.001$; two-sample $t$-test between visible and invisible condition, $t(8.167)=2.98, p=0.017)$ (Fig. $4 b)$. This reflects 

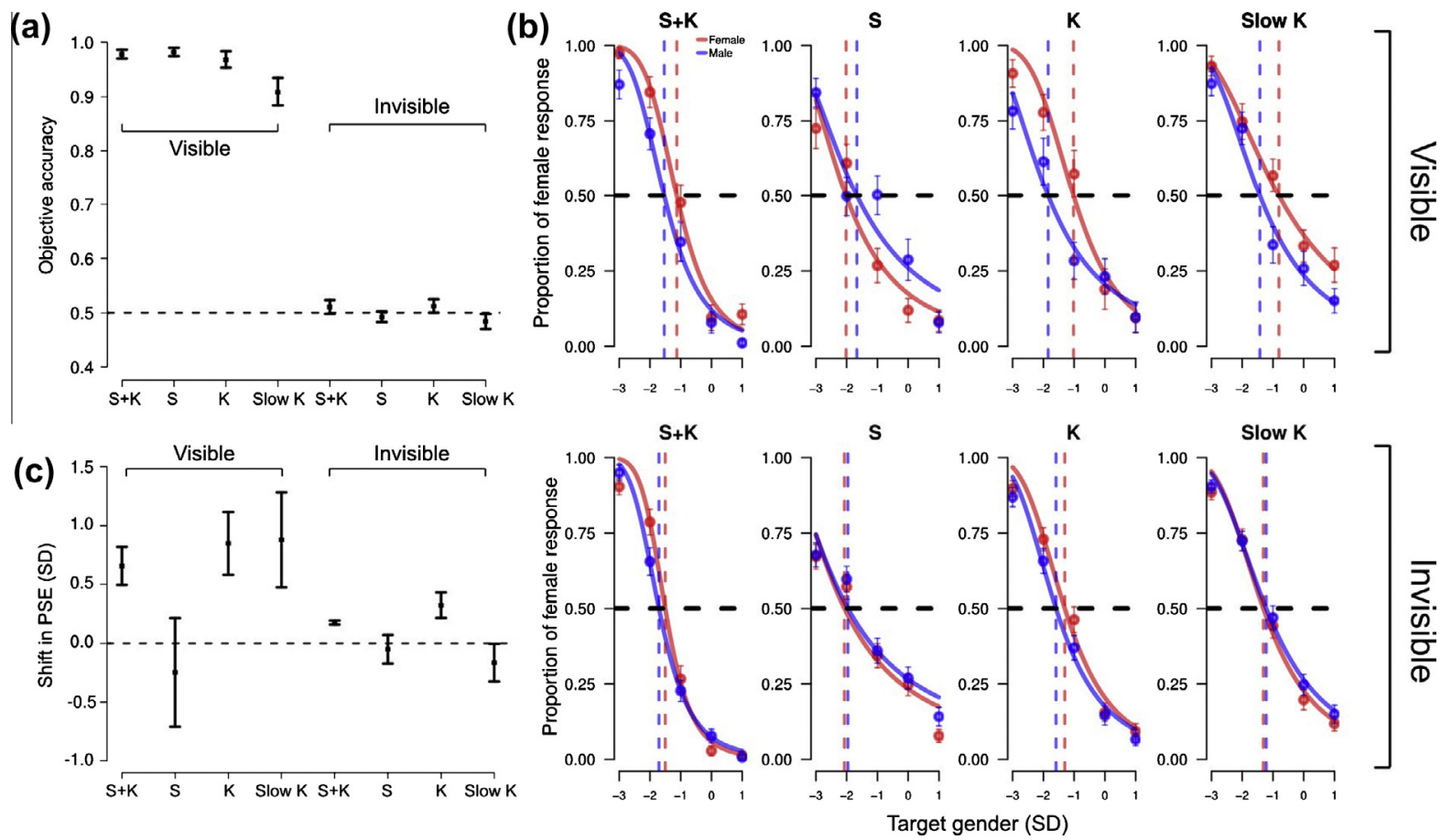

Fig. 4. Psychometric functions and shifts of perceptual equality. (a) Objective accuracy in the visible and invisible conditions (after excluding trials in which the adaptor was visible, see methods), for walkers defined by structural and kinematic cues ( $+\mathrm{K})$, structural cues only (S), kinematic cues only (K), and slow kinematic cues (Slow K). The horizontal dashed line corresponds to chance-level performance (i.e., 50\%, for 2 different adaptors). (b) Each graph represents the proportion of probes categorized as female and the estimated psychometric curve, after adaptation to a male (blue), or female (red) adaptor in the visible (top row) and invisible condition (bottom row). Vertical lines stand for the perceptual point of equality (i.e., a hypothetical probe categorized as a female with a probability of 0.5 ). Units are in standard deviation of gender (SD; see methods). c. Corresponding mean point of subjective equality shifts. Error bars correspond to SEM. (For interpretation of the references to color in this figure legend, the reader is referred to the web version of this article.)

an attractive, priming effect, whereby a neutral probe is perceived as a female after an exaggerated female adaptor, and vice versa. Due to the constraints imposed by CFS, the perceptual strength associated with the adaptor had to be much lower in our paradigm than in other studies measuring adaptation to visible point-light walkers (i.e., $3 \mathrm{~s}$ and 0.08 Michelson contrast, compared to $3-14 \mathrm{~s}$ and $\sim 1$ Michelson contrast in the study by Troje et al. (2006), $11.67 \mathrm{~s}$ and $\sim 1$ Michelson contrast in the study by Jordan et al. (2006)). We thought that this difference could explain why we found positive priming (i.e., a neutral probe is perceived as female after an exaggerated female adaptor, and vice versa) while these two other studies reported adaptation (i.e., a neutral probe is perceived as female after an exaggerated male adaptor, and vice versa). We validated this hypothesis by running a control experiment, in which a visible adaptor presented during $7 \mathrm{~s}$ at 0.08 Michelson contrast (i.e., with higher perceptual strength) exerted not an attractive, but a repulsive effect (effect $=-0.74$, $t(5)=-2.81, p=0.038$ ). This result is similar to previous studies documenting the relationship between visual adaptation and perceptual strength of orientation features (Faivre \& Kouider, 2011b), faces (Barbot \& Kouider, 2012), or translational motion (Kanai \& Verstraten, 2005).

When using adaptors containing only structural cues ( $3 \mathrm{~s}$ duration), no point of subjective equality shift was observed in the visible $($ effect $=-0.25, S E=0.46, t(7)=-0.54$, $p=0.61$ ) nor the invisible conditions (effect $=-0.05$, $S E=0.12, t(7)=-0.42, p=0.69)$. The absence of point of subjective equality shift questions the level at which spatial cues are encoded: possibly, walkers' gender is inferred from high-level post-perceptual processes, which are not at play when judging the gender of subsequent ambiguous probes. We could test this hypothesis by measuring the role of high-level cognitive functions in gender after-effects, relying notably on the application of attentional loads (e.g., see Bahrami, Carmel, Walsh, Rees, \& Lavie, 2008 for the role of attention in tilt after-effects). Another possible reason for the absence of effect is the uncertainty with which structurally defined adaptors were perceived, as revealed by the weak correlation between subjective visibility and objective accuracy in the visible condition (adjusted $R^{2}=0.22$, compared to values above 0.6 in the other conditions, see above).

By contrast, attractive point of subjective equality shifts were found from adaptors containing kinematic cues only, both in the visible (effect $=0.85, S E=0.27, t(6)=3.19$, $p=0.019$ ) and invisible conditions (effect $=0.32, S E=0.11$, $t(8)=2.97, p=0.018$; two-sample $t$-test between visible and invisible condition, $t(8.014)=1.83, p=0.105$ ). Whether temporal integration occurs over the entire dynamic structure of the point-light walkers (i.e., the successive postures of the whole walker), or only the positions of each individual dot is debated (Giese \& Poggio, 2003; Lange 
\& Lappe, 2006). As the posture in structurally-defined point-light walkers did not give rise to point of subjective equality shift, our results tend to support kinematics as the main property driving gender priming, in accordance with other studies (Giese \& Poggio, 2003; Mather \& Murdoch, 1994; Thurman \& Grossman, 2008; Troje, 2002). The importance of kinematic cues for carrying high-level features in biological motion is supported by a recent fMRI study. It showed that when rendered invisible by crowding, dynamic facial expressions (i.e., another kind of biological motion) are not processed through the ventral visual pathway, but only through the dorsal visual pathway which is usually associated with the processing of motion information (Faivre, Charron, Roux, Lehéricy, \& Kouider, 2012).

In order to examine the extent of temporal integration for biological motion, we then used adaptors defined by slower kinematics (one walking cycle in $3 \mathrm{~s}$ instead of $1 \mathrm{~s})$. In the visible condition, observers were still able to discriminate the gender of the adaptor with high accuracy $(90.9 \%, S E=2.52)$, suggesting that the slower kinematics we employed carried sufficient information for gender discrimination. By contrast, the proportion of trials in which observers had no experience of the adaptor was similar for slow and fast kinematics (respectively $81.8 \%$ and $88.4 \%$, Welch two sample $t$-test, $t(14.71)=-0.59$, $p=0.56)$. Furthermore, observers failed to discriminate the gender of the adaptor in the invisible condition $(51.24 \%, t(8)=1.00, p=0.34)$, with similar accuracy as for faster kinematics (Welch two sample t-test, $t(14.55)=1.27, p=0.22$ ). This suggests that CFS is equally potent at suppressing biological motion with fast and slow kinematics. When using these slower kinematics, we obtained a significant point of subjective equality shift in the visible condition (effect $=0.89, S E=0.36, t(9)=2.48$, $p=0.035$ ), but not in the invisible condition ( effect $=-0.17$, $t(9)=-1.02, p=0.33$; paired two-sample $t$-test between visible and invisible condition, $t(9)=2.64, p=0.025)$. In addition, we found a significant difference in the point of subjective equality shift between fast and slow kinematics in the invisible condition (Welch two sample $t$-test, $t(15.46)=2.51, p=0.024)$, but not in the visible condition (Welch two sample $t$-test, $t(14.42)=-0.06, p=0.95)$. This shows that the manipulation of stimulus visibility had a critical impact on the processing of stretched temporal structures. Overall, as for apparent motion, we conclude that biological motion defined over long periods of time is integrated only in the presence of perceptual awareness (Fig. 4c).

\section{Discussion}

Taken together, our results show clear evidence for the encoding of invisible apparent and biological motion, two temporal structures defined at distinct levels of visual processing.

It is of note that our conclusions stem from two paradigms that differ in several ways, including stimulus properties and measures of processing. First, apparent motion adaptors were presented for $20 \mathrm{~s}$, while biological motion adaptors were presented for $3 \mathrm{~s}$. We did not aim to equate the duration of apparent and biological motion adaptors, but rather used the maximal duration allowed for each stimulus to maximize the signal to noise ratio. Considering the high saliency induced by continuous motion, it is not surprising that this maximal duration was about an order of magnitude shorter for biological than for apparent motion ( $3 \mathrm{~s}$ vs. $20 \mathrm{~s}$ ). We found that long-lasting apparent motion adaptors induced repulsive effects (i.e., perceiving the probe as moving horizontally after a vertical adaptor, and vice versa), while biological motion adaptors induced attractive effects (i.e., perceiving the probe as a female after a female adaptor, and vice versa). Along this line, it has been shown that increasing the duration of invisible stimuli could reverse attractive into repulsive effects (Barbot \& Kouider, 2012; Faivre \& Kouider, 2011b; Kanai \& Verstraten, 2005). Beyond stimulus duration, the temporal dynamics that defined apparent and biological motion likewise differed: while the temporal structures carrying the direction of apparent motion ranged from $100 \mathrm{~ms}$ to 1200 ms (i.e., different ISIs between disks), those carrying the gender of point-light walkers ranged from $1 \mathrm{~s}$ (i.e., one walk cycle in the fast kinematic condition) to $3 \mathrm{~s}$ (i.e., one walk cycle in the slow kinematic condition). In the latter case, the critical information driving gender adaptation effects may be defined by smaller temporal structures (i.e., fractions of walk cycle).

Yet, it remains likely that the size of temporal structures inducing adaptation was smaller for apparent motion than for biological motion (i.e., 100 ms vs. 1 s), as it was shown between translational and biological motions in condition of full visibility (Neri, Morrone, \& Burr, 1997). It suggests that the neurons in the visual cortex involved in the temporal processing of biological motion (possibly in the superior temporal sulcus (Grossman et al., 2000) and extrastriate body area (Downing, Jiang, Shuman, \& Kanwisher, 2001)) contain larger temporal receptive fields (Hasson, Malach, \& Heeger, 2010) than those that compute apparent motion in MT/V5 and MST (Muckli et al., 2002). Finally, a last notable difference between the two experimental procedures is the way we measured the processing of apparent and biological motion adaptors: while we used continuous reports on a single bistable probe for apparent motion, we relied on psychometric responses to a set of ambiguous probes for biological motion.

Nevertheless, the conclusions we derived from these distinct paradigms are identical: compared to conditions of full visibility, only temporal structures defined over short periods of time were able to induce adaptation, both for apparent and biological motion. We consider two nonmutually exclusive interpretations for these results. First, assuming that all other parameters are kept constant, increasing the ISI of apparent motion or decreasing the speed of point-light walkers necessarily affects motion strength (i.e., the illusion of apparent motion decreases with longer ISI, ${ }^{1}$ and the point-light walkers appear less

\footnotetext{
${ }^{1}$ As a control experiment, we asked a group of 7 new observers to rate the strength of visible apparent motion from 1 to 4 for each ISI condition. We found that the illusion of apparent motion decreases linearly when ISI increases (Adjusted $R^{2}=0.29, p=0.002$ ).
} 
salient when walking slower, see Cutting \& Kozlowski, 1977). Therefore, assuming that the sensitivity to motion signals decreases without awareness, only short temporal structures containing strong motion signals are likely to give rise to adaptation. Alternatively, the same phenomenon can be explained in case weak motion signals are processed despite unawareness, but not maintained over time and therefore not integrated into a unified temporal structure (Blake \& Lee, 2005). Such shrinkage of temporal integration windows without awareness can explain our results independently of the sensitivity decrease described above. Let us examine the different aspects of our results arguing for one or the other interpretation.

First of all, in the visible conditions of all experiments, adaptors remained discriminated with very high accuracy no matter their kinematics. Thus, although differences between conditions may have remained unnoticed because of ceiling effects, we can assume that all adaptors carried sufficient information for conscious discrimination of apparent and biological motion. Conversely, we showed that CFS prevented awareness for all the different adaptors, ruling out the possibility that fast kinematics were more consciously accessed than slow kinematics. These two points confirm the basic assumptions that (1) information of direction (for apparent motion) and gender (for biological motion) was available in all visible conditions, and (2) that motion strength was not confounded with visibility in the invisible conditions. Furthermore, all the adaptation effects we found in the visible condition were independent of motion strength (i.e., ISI for apparent motion, and walking speed for biological motion), and adaptation to apparent motion was constant despite the linear decrease in the subjective motion strength. The fact that adaptation does not decrease with motion strength is probably due to the existence of a compressive nonlinearity, with adaptation response saturating at high motion strength (Blake, Tadin, Sobel, Raissian, \& Chong, 2006). Similarly, the fact that adaptation to visible and invisible apparent motion was of similar amplitude at high motion strength (i.e., ISI $=100 \mathrm{~ms}$ ) is likely due to response saturation, which in this case would conceal the decrease of sensitivity under CFS (the saturation of adaptation responses is unlikely in case of biological motion, since the effect amplitude differed between the visible and invisible condition). Crucially, a reduction of sensitivity to weaker signals under CFS would predict a linear decrease of adaptation with motion strength, consistent with the linear decrease of the apparent motion illusion. The fact that we found a all or none pattern (i.e., significant effect at $100 \mathrm{~ms}$, no effect beyond $100 \mathrm{~ms}$ ) makes this hypothesis less likely. On the contrary, the presence/absence of temporal integration coupled with the compressive nonlinearity of adaptation response predicts this all or none pattern: in case the signal is integrated into a motion percept, it gives rise to adaptation of large amplitudes due to response saturation; in case the signal is not integrated, adaptation does not occur, even though the motion strength is high. By contrast, we found that nonconscious gender priming was of smaller amplitude in the slow kinematics compared to the fast kinematics condition (in accordance with the fact that slow point-light walkers were discriminated with lower accuracy in the conscious condition, see results). Thus, although our results favor a shrinkage of temporal integration windows as the most probable explanation of our results, a conjoint decrease in sensitivity to motion signals may coexist. Future experiments with finer temporal sampling, or experiments compensating the decrease of motion strength with non-temporal factors (e.g., spatial configuration of motion stimuli, or attentional amplification, see Lu \& Sperling, 1995), will be helpful to disentangle the respective influence of motion signal strength and temporal integration during the coding of temporal structures.

The limit of nonconscious temporal integration can be accounted for by two qualitatively distinct theoretical frameworks of consciousness. At an abstract level, the integrated information theory states that perceptual awareness depends on the system's ability to generate information as a whole (i.e., reducing uncertainty by processing specific physical features), above and beyond information generated by the sum of its parts (Tononi, 2010). While the theory does not directly take into account the processing of temporal structures, it seems plausible that within this framework, the consciousness level of a system should co-vary with its ability to integrate together events that are far apart in time. Hence, within this framework, the shrinking of temporal integration for motion processing is expected. Based on neurocognitive evidence, the global neuronal workspace model states that conscious access to a feature (e.g., motion) is associated with the ignition of recurrent neural activity between sensory regions and a fronto-parietal network (Dehaene \& Changeux, 2011). This model makes the prediction that consciousness is required for specific cognitive tasks, including those for which activity must be amplified and maintained over a sufficient duration for it to become accessible to multiple other processors. Previous results suggest that subliminal priming typically becomes undetectable after a few hundreds of milliseconds (e.g., Mattler, 2005), and that the chaining of cognitive operations is not possible without awareness (Sackur \& Dehaene, 2009). These two examples involve all or none criteria, according to which a function is enabled in the presence - but not the absence - of awareness. In comparison, we argue that estimating quantitatively the extent of temporal integration windows for visible and invisible stimuli is more powerful in detailing the differences between conscious and nonconscious vision.

At the neural level, recurrent cortical networks are good candidates to explain how consciously visible signals are maintained over long durations. Indeed, such networks are considered as a potential neural correlate of consciousness (Lamme \& Roelfsema, 2000), and have been shown to enable persistent neural activity and temporal integration (Wang, 2002). According to this view, the limit of nonconscious temporal integration may reflect the maximal time during which neural signals carrying the properties of a temporal structure are sustained when propagated in a feed-forward manner. Conversely, the ignition of neural recurrent activity associated with consciousness would extend this time, resulting in larger temporal integration 
windows. Another neural mechanism that is commonly associated with consciousness and integration of multiple component processes is temporal binding, by which neuronal discharges are synchronized with a precision in the millisecond range (Engel \& Singer, 2001). Using electroencephalography, it has been shown that when two stimuli are integrated into a single percept, the eventrelated potentials mostly originate from the first stimulus, the second one exerting an influence mostly in terms of amplitude modulation (Akyürek \& Meijerink, 2012). Accordingly, this modulation might reflect ongoing neural synchronization of the two successive stimuli, and therefore their temporal binding. Together with the behavioral effects we report, functional evidence for neural recurrent activity and neural synchronization should help delimiting conscious and nonconscious temporal integration.

Besides stimulus awareness, attentional amplification is likely to play a role during temporal integration. Notably, attending to a brief stimulus is known to increase its perceived duration (Yeshurun \& Marom, 2008), while attending to two successive contours is known to increase the duration over which they can be combined into a single unified percept (Visser \& Enns, 2001). Accordingly, we predict that attending to apparent motion stimuli should extend the duration of ISIs over which adaptation effects occur, by increasing the activations elicited by the disks, or decreasing their temporal decay. Similarly, attention should enhance the capacity to extract gender information from slow kinematics in point-light walkers (see Thompson \& Parasuraman, 2012 for a review of attentional effects on the perception of biological motion). Conversely, attentional amplification is also known to degrade temporal resolution, which is the capacity to discriminate the transition of static features within a temporal structure (Yeshurun \& Levy, 2003). For instance, attention has detrimental effects on the discrimination of short-range apparent motion, defined by short ISIs or spatial displacement between disks below $1^{\circ}$ (Yeshurun $\&$ Hein, 2011). As attention and consciousness are intricate phenomena (Cohen, Cavanagh, Chun, \& Nakayama, 2012; Koch \& Tsuchiya, 2007; Lamme, 2003) it would be interesting to assess their respective influences on temporal integration and temporal resolution. Notably, it will be important to test whether the decrease of integration during nonconscious or unattended vision generalizes to spatial features (Mudrik, Breska, Lamy, \& Deouell, 2011; Faivre \& Koch, in press), temporal features other than motion (e.g., sequentiality, causality), and to masking techniques other than CFS (e.g., gaze-contingent crowding, see Faivre \& Kouider, 2011a; Kouider, Berthet, \& Faivre, 2011). This will provide objective measures to quantify perceptual awareness at the behavioral level, and, help to delimit the fundamental links between time, attention and consciousness.

\section{Authorship}

NF and CK contributed to the study design. Testing, data collection and data analysis were performed by NF. NF and $\mathrm{CK}$ wrote the paper. All authors approved the final version of the paper for submission.

\section{Acknowledgments}

This research was supported by G. Harold \& Leila Y. Mathers Charitable Foundation. N.F was supported by the Fyssen Foundation. The authors are very grateful to Niko Troje for providing the point-light walkers stimuli, and to Liad Mudrik and Jeroen van Boxtel for their help with the manuscript. The authors declare no competing financial interests.

\section{References}

Akyürek, E. G., \& Meijerink, S. K. (2012). The deployment of visual attention during temporal integration: An electrophysiological investigation. Psychophysiology, 49, 885-898. http://dx.doi.org/ 10.1111/j.1469-8986.2012.01380.x.

Anstis, S., Giaschi, D., \& Cogan, A. I. (1985). Adaptation to apparent motion. Vision Research, 25, 1051-1062.

Anstis, S. M. (1980). The perception of apparent movement. Philosophical Transactions of the Royal Society of London. Series B, Biological sciences, 290, 153-168.

Bahrami, B., Carmel, D., Walsh, V., Rees, G., \& Lavie, N. (2008). Unconscious orientation processing depends on perceptual load. Journal of Vision, 8(3), 1-10. http://dx.doi.org/10.1167/8.3.12. http:// journalofvision.org/8/3/12/.

Barbot, A., \& Kouider, S. (2012). Longer is not better: Nonconscious overstimulation reverses priming influences under interocular suppression. Attention, Perception, E Psychophysics, 74, 174-184.

Blake, R., Ahlström, U., \& Alais, D. (1999). Perceptual priming by invisible motion. Psychological Science, 10, 145-150.

Blake, R., \& Lee, S. H. (2005). The role of temporal structure in human vision. Behavioral and Cognitive Neuroscience Reviews, 4, 21-42.

Blake, R., Tadin, D., Sobel, K. V., Raissian, T. A., \& Chong, S. C. (2006). Strength of early visual adaptation depends on visual awareness. Proceedings of the National Academy of Sciences of the United States of America, 103, 4783-4788.

Brainard, D. H. (1997). The psychophysics toolbox. Spatial Vision, 10, 433-436.

Cohen, M. A., Cavanagh, P., Chun, M. M., \& Nakayama, K. (2012). The attentional requirements of consciousness. Trends in Cognitive Sciences., 16, 411-417.

Cutting, J. E., \& Kozlowski, L. T. (1977). Recognizing friends by their walk: Gait perception without familiarity cues. Bulletin of the Psychonomic Society, 9, 353-356.

Dawson, M. R. (1991). The how and why of what went where in apparent motion: Modeling solutions to the motion correspondence problem. Psychological Review, 98, 569-603.

Dehaene, S., \& Changeux, J. P. (2011). Experimental and theoretical approaches to conscious processing. Neuron, 70, 200-227.

Downing, P. E., Jiang, Y., Shuman, M., \& Kanwisher, N. A. (2001). Cortical area selective for visual processing of the human body. Science, 293, $2470-2473$.

Engel, A. K., \& Singer, W. (2001). Temporal binding and the neural correlates of sensory awareness. Trends in cognitive sciences, 5(1), $16-25$.

Faivre, N., \& Kouider, S. (2011a). Increased sensory evidence reverses nonconscious priming during crowding. Journal of Vision, 11(13:1), $1-13$.

Faivre, N., \& Kouider, S. (2011b). Multi-feature objects elicit nonconscious priming despite crowding. Journal of Vision, 11(3:), 1-10.

Faivre, N. \& Koch, C., Inferring the direction of implied motion depends on visual awareness, Journal of Vision, in press.

Faivre, N., Charron, S., Roux, P., Lehéricy, S., \& Kouider, S. (2012). Nonconscious emotional processing involves distinct neural pathways for pictures and videos. Neuropsychologia, 50(14), 3736-3744.

Geisler, W. S. (1999). Motion streaks provide a spatial code for motion direction. Nature, 400, 65-69.

Giese, M. A., \& Poggio, T. (2003). Neural mechanisms for the recognition of biological movements. Nature Reviews Neuroscience, 4, 179-192.

Grossman, E., Donnelly, M., Price, R., Pickens, D., Morgan, V., Neighbor, G., et al. (2000). Brain areas involved in perception of biological motion. Journal of Cognitive Neuroscience, 12, 711-720.

Hasson, U., Malach, R., \& Heeger, D. J. (2010). Reliability of cortical activity during natural stimulation. Trends in Cognitive Sciences, 14, 40-48. 
Johansson, G. (1973). Visual perception of biological motion and a model for its analysis. Perception $\mathcal{E}$ Psychophysics, 14, 201-211.

Jordan, H., Fallah, M., \& Stoner, G. R. (2006). Adaptation of gender derived from biological motion. Nature Neuroscience, 9, 738-739.

Kanai, R., \& Verstraten, F. A. J. (2005). Perceptual manifestations of fast neural plasticity: Motion priming, rapid motion aftereffect and perceptual sensitization. Vision Research, 45, 3109-3116.

Koch, C., \& Tsuchiya, N. (2007). Attention and consciousness: Two distinct brain processes. Trends in cognitive sciences, 11(1), 16-22.

Kohler, A., Haddad, L., Singer, W., \& Muckli, L. (2008). Deciding what to see: The role of intention and attention in the perception of apparent motion. Vision Research, 48, 1096-1106.

Kouider, S., Berthet, V., \& Faivre, N. (2011). Preference is biased by crowded facial expressions. Psychological Science, 22, 184-189.

Kruse, P., Stadler, M., \& Wehner, T. (1986). Direction and frequency specific processing in the perception of long-range apparent movement. Vision Research, 26, 327-335.

Lamme, V. A., \& Roelfsema, P. R. (2000). The distinct modes of vision offered by feedforward and recurrent processing. Trends in Neurosciences, 23(11), 571-579.

Lamme, V. A. (2003). Why visual attention and awareness are different Trends in Cognitive Sciences, 7, 12-18.

Lange, J., \& Lappe, M. (2006). A model of biological motion perception from configural form cues. Journal of Neuroscience, 26, 2894-2906.

Lin, Z., \& He, S. (2009). Seeing the invisible: The scope and limits of unconscious processing in binocular rivalry. Progress in Neurobiology, 87, 195-211.

Lu, Z. L., \& Sperling, G. (1995). Attention-generated apparent motion. Nature, 377(6546), 237-239.

Mather, G., \& Murdoch, L. (1994). Gender discrimination in biological motion displays based on dynamic cues. Proceedings of the Royal Society of London: Series B, Biological Sciences, 258, 273-279.

Mattler, U. (2005). Inhibition and decay of motor and nonmotor priming. Perception E Psychophysics, 67, 285-300.

Muckli, L., Kriegeskorte, N., Lanfermann, H., Zanella, F. E., Singer, W., \& Goebel, R. (2002). Apparent motion: Event-related functional magnetic resonance imaging of perceptual switches and States. Journal of Neuroscience, 22. RC219.

Mudrik, L., Breska, A., Lamy, D., \& Deouell, L. Y. (2011). Integration Without Awareness Expanding the Limits of Unconscious Processing. Psychological Science, 22(6), 764-770.

Neri, P., Morrone, M. C., \& Burr, D. C. (1997). Seeing biological motion. Nature, 395(894-896), 1998.

Pelli, D. (1997). The VideoToolbox software for visual psychophysics: Transforming numbers into movies. Spatial Vision, 10, 437-442.

R Core Team (2013). R: A language and environment for statistical computing. R Foundation for Statistical Computing, Vienna, Austria. http://www.R-project.org/.
Rajimehr, R., Vaziri-Pashkam, M., Afraz, S. R., \& Esteky, H. (2004) Adaptation to apparent motion in crowding condition. Vision Research, 44, 925-993.

Ramachandran, V. S. (1975). Suppression of apparent movement during binocular rivalry. Nature, 256, 122-123.

Ramsøy, T., \& Overgaard, M. (2004). Introspection and subliminal perception. Phenomenology and the Cognitive Sciences, 3(1), 1-23.

Sackur, J., \& Dehaene, S. (2009). The cognitive architecture for chaining of two mental operations. Cognition, 111(2), 187-211.

Thompson, J., \& Parasuraman, R. (2012). Attention, biological motion, and action recognition. Neuroimage, 59(1), 4-13.

Thurman, S.M., \& Grossman, E.D. (2008). Temporal “Bubbles” reveal key features for point-light biological motion.

Tononi, G. (2010). Information integration: Its relevance to brain function and consciousness. Archives Italiennes de Biologie, 148, 299-322.

Troje, N. F. (2002). Decomposing biological motion: A framework for analysis and synthesis of human gait patterns. Journal of Vision, 2, 371-387.

Troje, N. F., Sadr, J., Geyer, H., \& Nakayama, K. (2006). Adaptation aftereffects in the perception of gender from biological motion. Journal of Vision, 6, 850-857.

Troje, N. F. (2008). Retrieving information from human movement patterns. In T. F. Shipley \& J. M. Zacks (Eds.), Understanding events: How humans see. Represent, and act on events (pp. 308-334). Oxford University Press.

Tsuchiya, N., \& Koch, C. (2005). Continuous flash suppression reduces negative afterimages. Nature Neuroscience, 8, 1096-1101.

Tsuchiya, N., Koch, C., Gilroy, L. A., \& Blake, R. (2006). Depth of interocular suppression associated with continuous flash suppression, flash suppression, and binocular rivalry. Journal of Vision, 6, 1068-1078.

Visser, T. A. W., \& Enns, J. T. (2001). The role of attention in temporal integration. Perception, 30(2), 135-145.

Wang, X.-J. (2002). Probabilistic decision making by slow reverberation in cortical circuits. Neuron, 36, 955-968.

Whelan, R. (2010). Effective analysis of reaction time data. The Psychological Record, 58, 9.

Wiesenfelder, H., \& Blake, R. (1991). Apparent motion can survive binocular rivalry suppression. Vision Research, 31, 1589-1599.

Yeshurun, Y., \& Levy, L. (2003). Transient spatial attention degrades temporal resolution. Psychological Science, 14(3), 225-231.

Yeshurun, Y., \& Marom, G. (2008). Transient spatial attention and the perceived duration of brief visual events. Visual Cognition, 16(6) 826-848.

Yeshurun, Y., \& Hein, E. (2011). Transient attention degrades perceived apparent motion. Perception, 40(8), 905-918. 